\title{
Panel Discussion IV
}

\author{
Panel: F. Allard, A. Batten, E. Budding, E. Devinney, P. Eggleton, A. Hatzes, \\ I. Hubeny, W. Kley, H. Lammer, A. Linnell, V. Trimble, and R. E. Wilson
}

\section{Discussion}

I. HubenY: Welcome to the last panel meeting. We invite general comments either from the audience or from the panelists.

V. Trimble: Well, Mercedes started us with a vocabulary item and I think I would like to end with a vocabulary item. When they were first discovered, we called them 'extra solar system planets' which was descriptive and fine, but it's just rather cumbersome. At some point they became 'extra solar planets.' Now I have never seen a planet inside the Sun. And therefore 'extrasolar' is not a good descriptor. 'Exoplanets' is OK, but now that there are so many of them that perhaps they are simply 'the planets.' When you want to specialize to ours, you could say 'solar system planets.' Think how much ink it would save.

W. KLEY: We heard today again in the talk by Ian Dobbs-Dixon that there is a strong similarity between exoplanets and secondary stars in close binaries. The only difference he said is in the chemistry, but I think the disk is also there. Nevertheless, these suggestions are very similar and I think this is a good connection with the theme of the conference: planets and binaries. They are the same thing, and it is clear that the planet is also a secondary object, like the secondary star in a binary. But, one thing which came to my mind yesterday is that when you're looking at the light curves that you analyze for the exoplanets, there are lots of different light curve modeling procedures around. So the question came up, these models that you developed to analyze binary eclipse light curves, are they suitable for planets or are we using different models, and what is the relation between them? Are they completely independent? You find on planets these displaced hot spots and so on, I assume all these results must have been familiar to the binary synthesis light curve modelers. So, I think there is a good connection between binaries and planets in several aspects.

O. De Marco: Dr. Kley took the words from my mouth. I'll put an example to this in the other direction. I'm interested in irradiated binaries that come out of common envelopes and then become central stars or planetary nebula. There are very strong irradiation effects, and we model them with some Wilson-Devinney type codes. Now, one thing that worried me a lot is the transfer radiation from one side to the other, and some of these systems are tidally locked, and some probably are not yet. There should be in theory very different light curve properties and, now that Kepler is taking some data of these systems, I don't think we can avoid thinking of these effects of radiation transfer. Now, the 'Hot Jupiter' people, like Ian, did a great job of that; but capacitance will be very different so radiation transfer will be different. So, I would love to have a way, or for somebody to do this for me, to give us a tool in the Wilson-Devinney code with some approximation for these effects that can help with this particular problem. I think the pieces are there. 
R. WILSON: I had not even realized the program was being used all that much for planets. For most purposes, it appears to me that there are adequate programs around that the exoplanet community have written that handle the job, since the stars are basically spherical in most cases. In many aspects the problems are simpler, but there are some aspects in which it's more complicated that we just mentioned. The heating problem, reflection, and so on, and Jan Budaj's paper at this meeting on the reflection effect and the other things you've mentioned about radiation coming from heat transfer around the back, those are not in the binary star programs now. So, there's some work to do for the binary star community.

I. Hubeny: I think convergence of modeling approaches is also being seen on the level of synthetic codes, like BINSYN that Al Linnell mentioned earlier, namely spectrum synthesis. It's already being done for close binaries using the same basic codes. TLUSTY was originally a stellar code and now it is being used for planets as well. So from that point of view, there is certainly convergence, and there are those synthetic models not specifically tailored for light curves but for predicting spectra as a function of phase, they certainly exist. As far as spectra are concerned, there is more progress now in planets than in stars.

A. BAtten: I would like to make a very general comment that the powerpoint presentations worked very well at this conference. There used to be many problems fitting visitor's laptop computers to resident projection systems, and these deterred me from using powerpoint myself until less than a year ago. The problems were not in evidence here and I particularly enjoyed the animations that illustrated several talks, including two this morning. So, powerpoint presentations seem to have come of age.

A story: During coffee, Ed Guinan and I were discussing the question of whether any star was truly constant in velocity, luminosity, etc.. This reminded me that R. M. Petrie, my boss in my early days at the Dominion Astrophysical Observatory, used to quote H. D. Curtis (of the Shapley-Curtis debate). Curtis was very fond of saying: "There is more joy in Heaven over one star that is found to be constant than over ninety-and-nine that are known to be variable!"

A. HAtzes: I find that as I get older, I like to get my pearls of wisdom, whether they're wisdom or not. I noticed that there are a lot of young people here and the topic of this conference was about essential tools. I also saw these beautiful codes that people use for modelling the stellar atmosphere have come a long way. But, as a warning to the young people here: there's a tendency to use things that are black boxes. There can be a real danger in that. So, I encourage you to understand the physics behind these black boxes. Don't just use them blindly. Understand how the code works and even better yet, look into the code. And, I give you two examples from my own experience: When we were looking for planets and finding them with Bill Cochran before it was fashionable and we needed to compute orbits, our colleagues in Texas said, "Oh there's a spectroscopic binary orbit, it works, use these!" So we used them and we started to get eccentric orbits and it was producing garbage. We thought, "Well, let's look into the code." Well it's good we looked into the code (it was in FORTRAN, so only dinosaurs like me could read the code), because there was a comment: "Doesn't work for eccentricities above 0.2" So, it was completely invalid to use it!

Another more serious one. My colleague Heike Rauer does work on planetary atmospheres. She got a standard code, was using it and seeing some irregularities, so she decided to look into the code and there were some parameters that went negative which 
shouldn't. These were physical variables like temperature that should not go negative. What does the code do? It sets the values to zero and merrily goes on and produces an answer. So be careful. You don't want to base your career on someone else's mistakes. They're very clever people but I'm still finding bugs in my own code, years after the fact. So user beware.

E. Budding: Continuing from the comment of Dr. Hatzes, I would like to refer to the subject of the Roche lobe, which has been mentioned a number of times at this meeting. Incidentally, Edouard Albert Roche lived in the nineteenth century. Alan here will recall that the person who introduced this term and provided a lot of work for its early development was very sensitive to its use. He would frequently recall that the Roche contours were a very artificial device, relating to equipotential surfaces, i.e. where matter was at zero velocity, and in a situation with two mass points moving in circular orbits, with rigidly rotating massless envelopes. All of these restrictions have been broken in the contexts in which these formulae for the potential were being applied, so it is well to remember that such theoretical modellings, detailed as they may be, are approximations.

On the other hand, as we have seen, there are also accumulating masses of detailed data, but they also still contain errors and have a finite information content. The appropriate matching of these data by suitable models has been the challenge before us, but this matching process requires us to keep in mind the key issues of model adequacy and determinacy.

E. Devinney: Maybe it's because of my age and generation, but I was thinking about how rich nature is as we see the phenomena we've discussed at this meeting. Musing about this to myself in these last few moments and thinking probably there are a lot of us older persons here who, when you talk about exoplanets or when exoplanets were first found, said, "This is incredible." But, maybe younger people are thinking "Yeah, it's interesting." And probably in another ten years, people will just think "Oh, yeah, exoplanets." So, it is great to be at this stage where we can think about these things with some awe and amazement.

I. Hubeny: Thank you. With those wise words we will end our panel discussion. 